Supplementary Information for Langmuir

\title{
Pegylated Metal-Phenolic Networks for Antimicrobial and Antifouling Properties
}

Hsiao-Tung Zheng, ${ }^{\dagger}$ Hoang Linh Bui, ${ }^{\dagger}$ Subhendu Chakroborty, ${ }^{\dagger}$ Yi Wang, ${ }^{\uparrow}, *$ Chun-Jen Huang ${ }^{\dagger}$, $\#, \&, *$

${ }^{\dagger}$ Department of Biomedical Sciences and Engineering, ${ }^{*}$ Department of Chemical and Materials Engineering, National Central University, Jhong-Li, Taoyuan 320, Taiwan. ${ }^{\text {R }}$ \&D Center for Membrane Technology, Chung Yuan Christian University, Chungli, Taoyuan, Taiwan. "School of Ophthalmology \& Optometry, Eye Hospital, School of Biomedical Engineering, Wenzhou Medical University, PR China; Wenzhou Institute, University of Chinese Academy of Sciences, Wenzhou 325001, PR China

*Corresponding author: cjhuang@ncu.edu.tw (C.-J.H.); wangyi@wibe.ac.cn (Y. W.) 


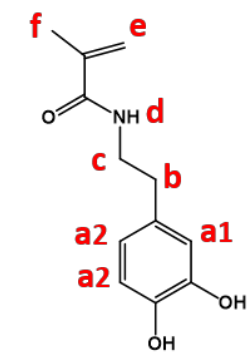

\section{d-DMSO}
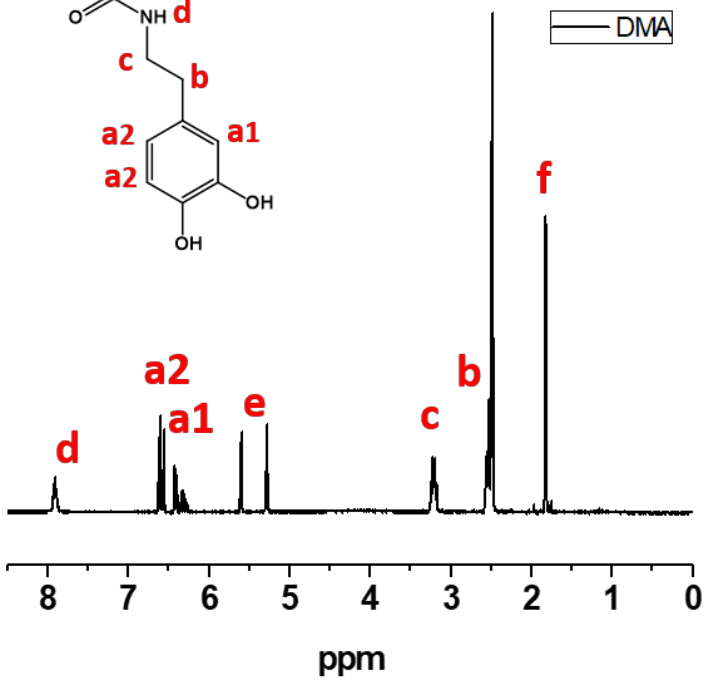

Figure S1. 1H NMR spectrum of DMA.
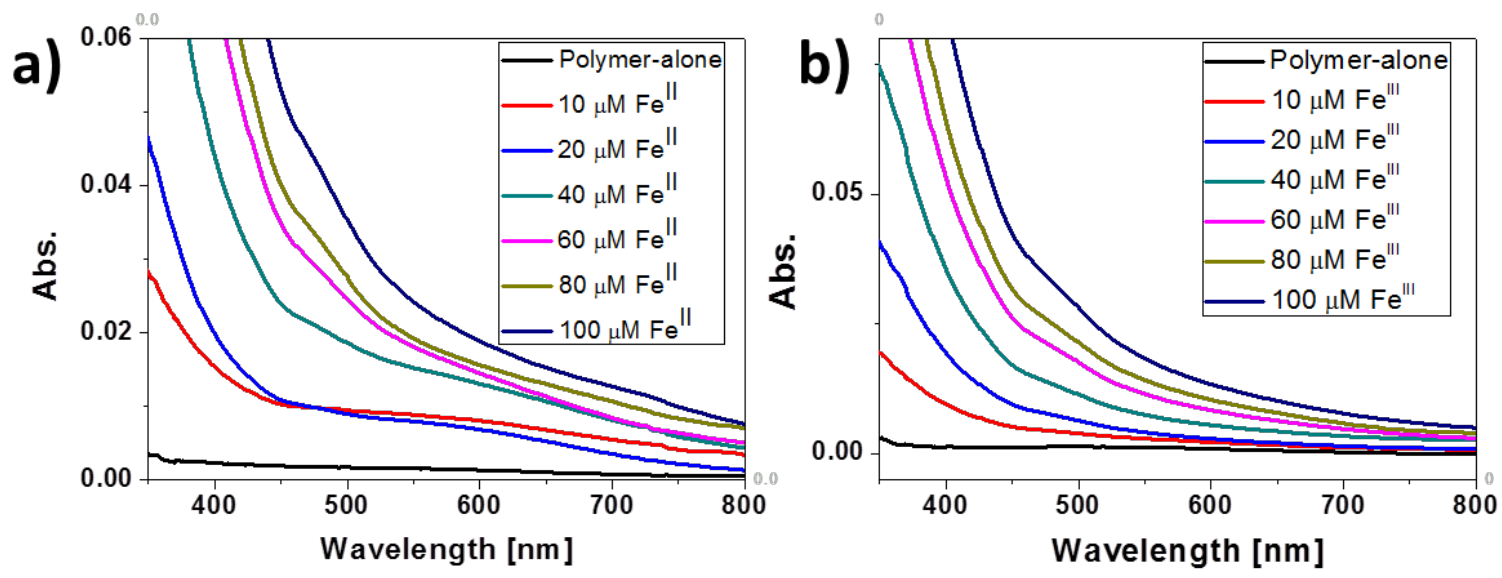

Figure S2. UV-vis spectroscopy measurements for the formation of the coordination structures between $\mathrm{p}\left(\mathrm{PEGMA} \mathrm{A}_{8}-\mathrm{CO}-\mathrm{DMA}_{2}\right)$ and metal ions of $\mathrm{Fe}$ "I $(\mathrm{a})$ and $\mathrm{Fe} \mathrm{III}^{\mathrm{II \prime}}(\mathrm{b})$. The photometer was used with a scan range from 450 to $800 \mathrm{~nm}$. The polymer solution was prepared at a concentration of $0.001 \% \mathrm{w} / \mathrm{v}$ in $\mathrm{pH} 8.5$ tris buffer and the Fe solution was mixed with the p(PEGMA-Co-DMA) solution to attain a series of metal ion concentrations from 10 to $100 \mu \mathrm{M}$. 

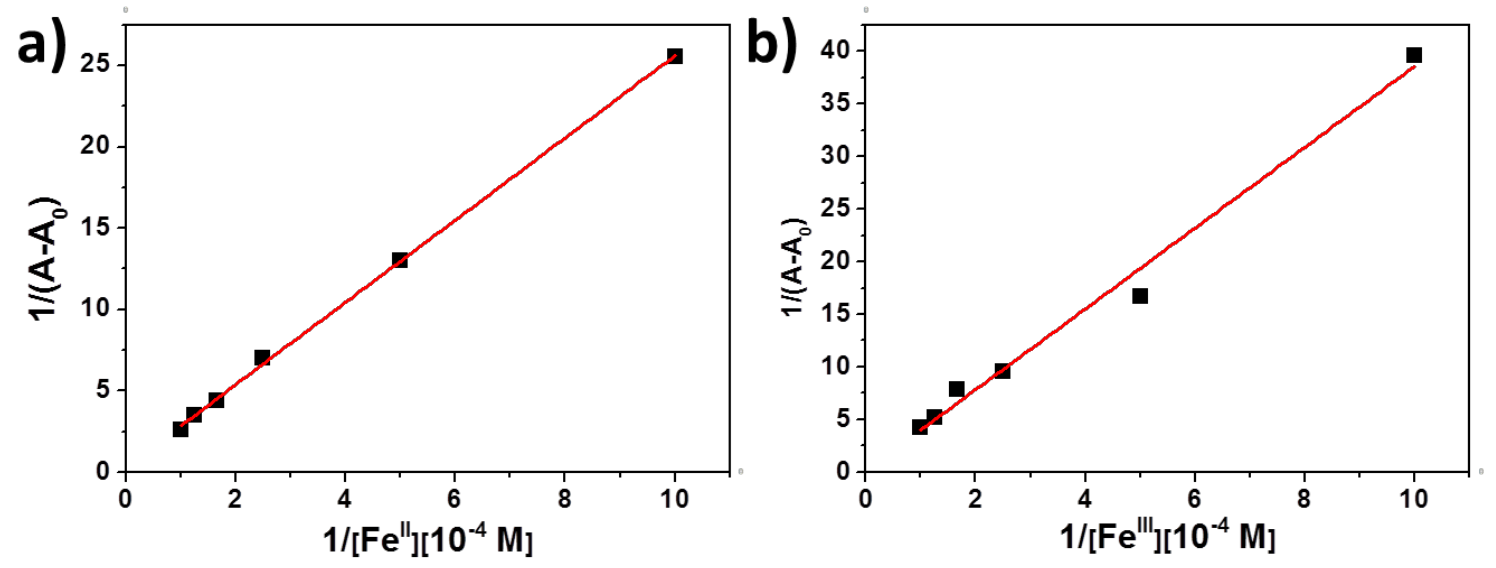

Figure S3. Klotz plots for binding constant of $\mathrm{Fe}^{\text {II }}(\mathrm{a})$ and Fe $\mathrm{III}^{\mathrm{II}}(\mathrm{b})$ with $\mathrm{p}\left(\mathrm{PEGMA}_{8}-\mathrm{Co}-\mathrm{DMA}_{2}\right)$. The analysis for the absorbance at a wavelength of $288 \mathrm{~nm}$ was conducted.

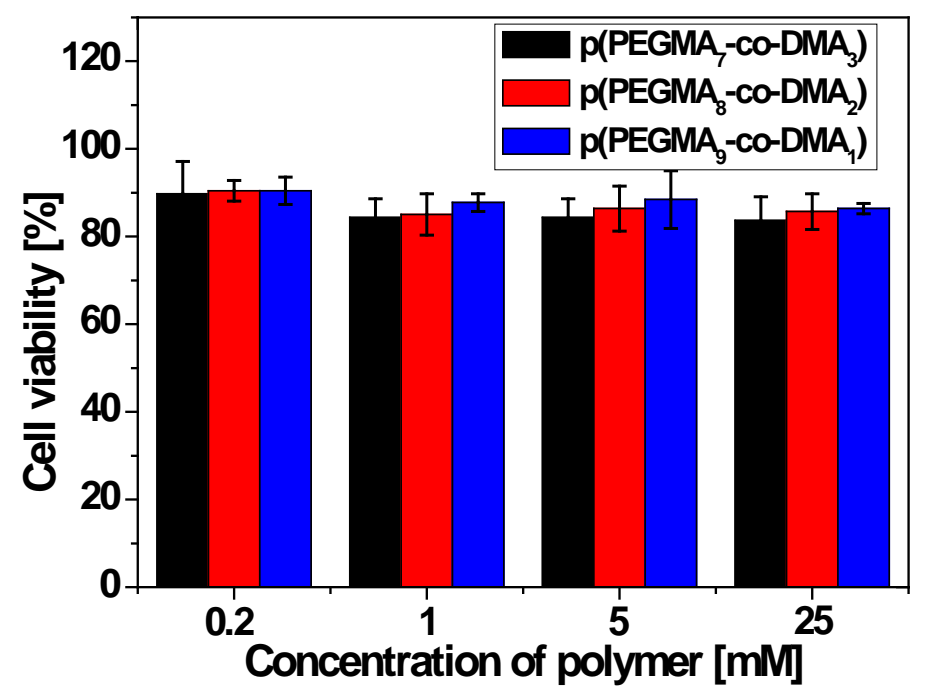

Figure S4. The cell viability of NIH 3T3 fibroblasts for examining the cytotoxicity of p(PEGMA-co-DMA) in a range of concentrations from 0.2 to $25 \mathrm{mM}$ in serum-free culture medium. The cell viability was calculated with respect to the cell culture without any treatment. In the experiments, each samples were conducted in triplicate $(n=3)$. 

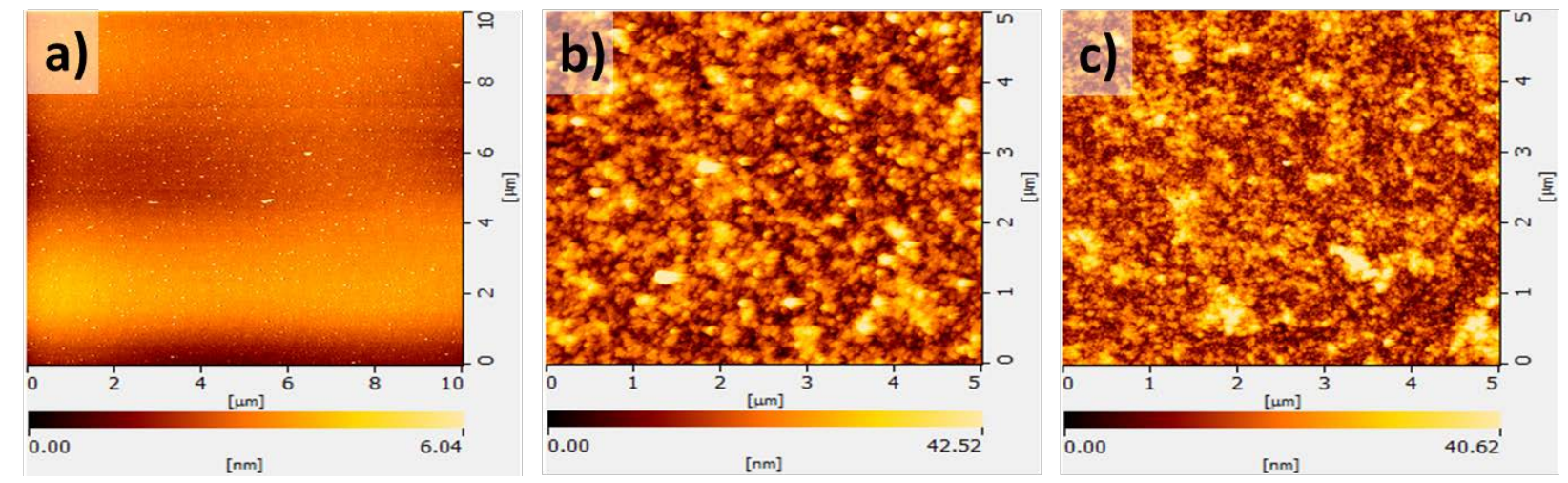

Figure S5. AFM topography images of bare (a), 3-Fe" (b) and 3-Fe'III (c).

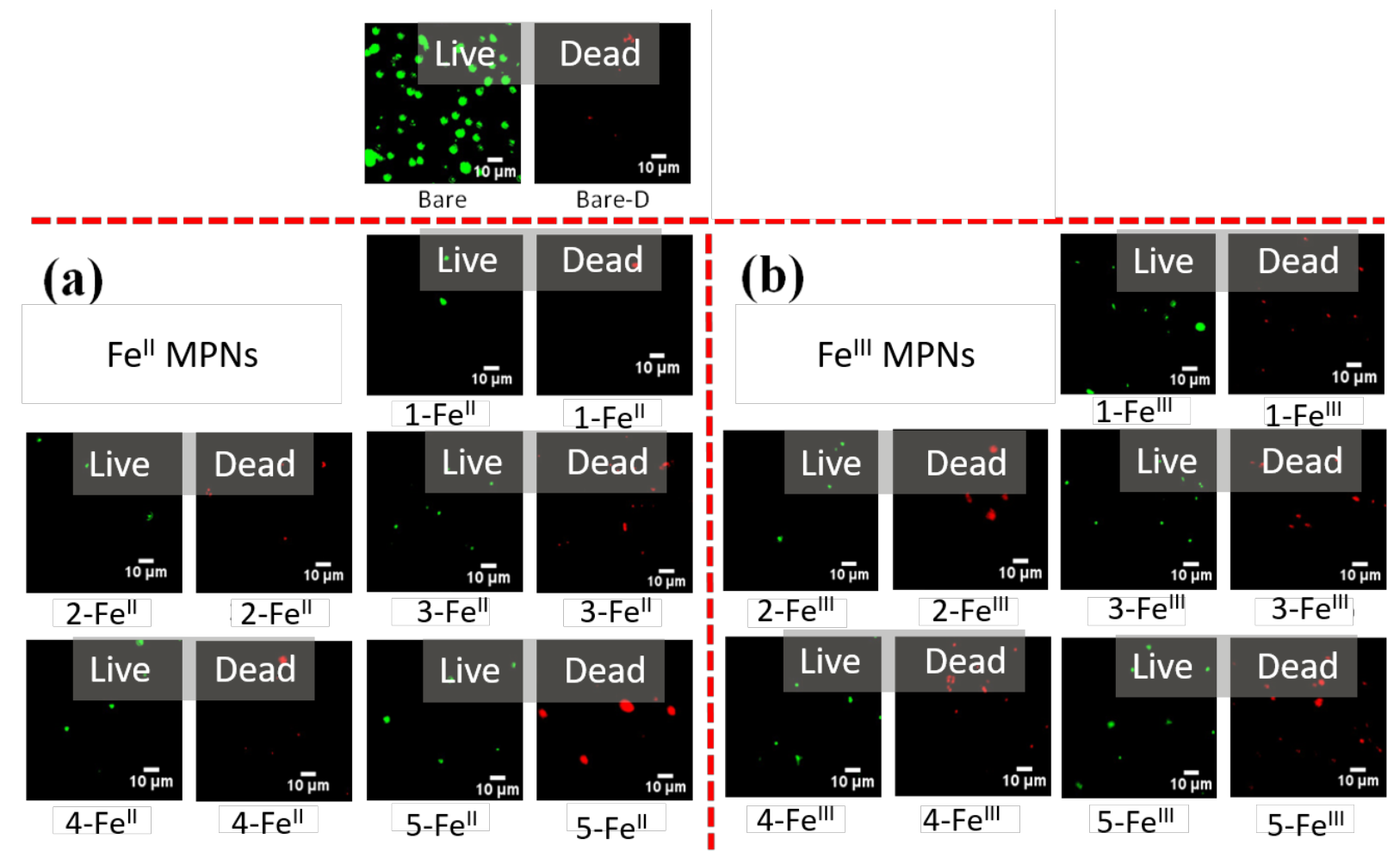

Figure S6. Fluorescence images for live and dead E. coli on samples. 


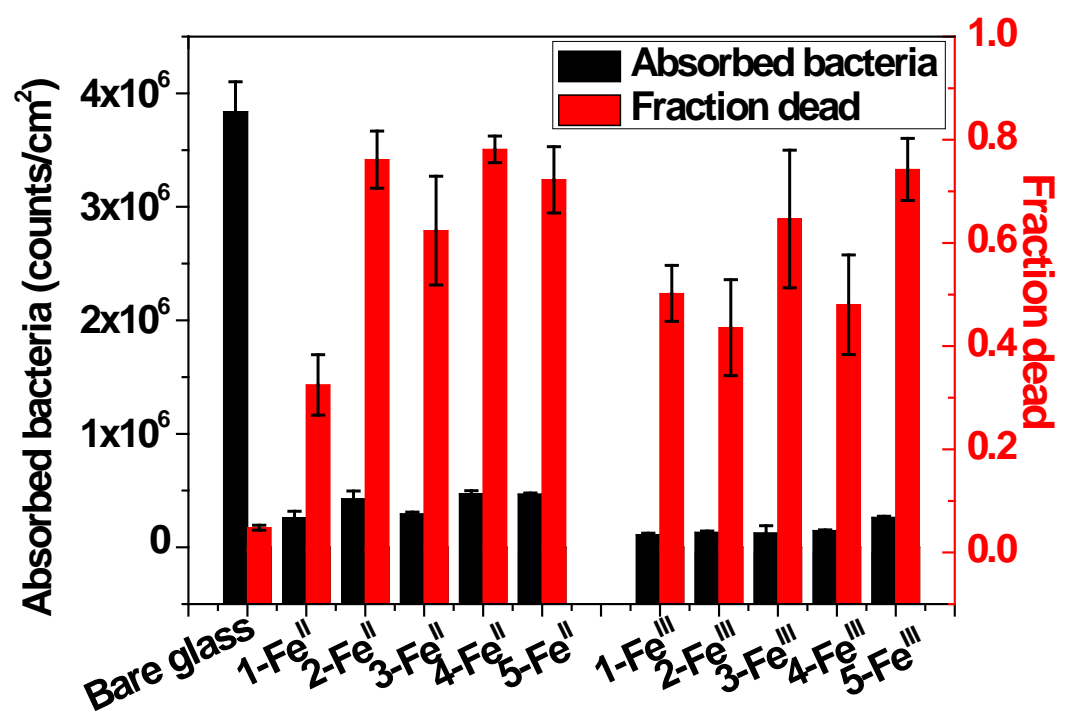

Figure S7. Total number of absorbed bacteria counts per area (black bars) and fraction of bacteria dead (red bars) for S. epidermidis on bare glass and MPN-coated substrates. Values in a and b represent the mean and the standard deviation $(n=3)$.
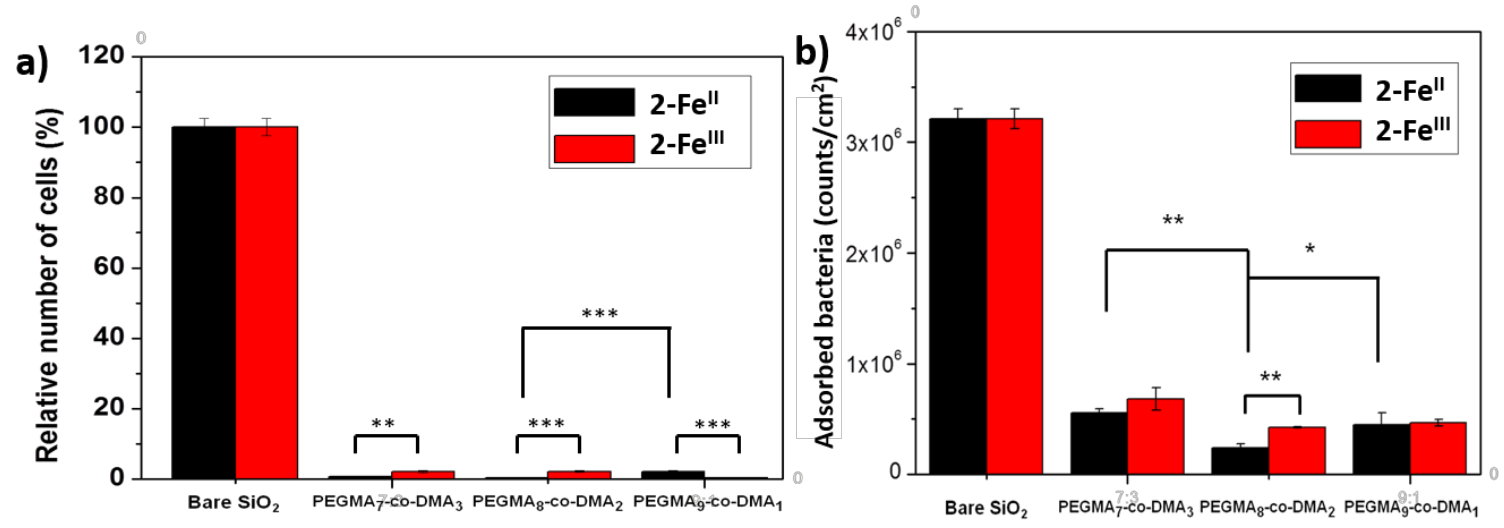

Figure S8. a) Relative numbers of NIH-3T3 fibroblasts on MPN coatings with respect to bare glass. The MPN coatings were prepared by co-deposition of $p\left(P E G M A_{7}-c_{0}-D M A_{3}\right), p\left(P E G M A_{8}-{ }^{-C o}-D_{M A}\right)$ and p (PEGMAg-Co-DMA ${ }_{1}$ ) with metal ions of Fe" or Fe"ll with deposition number of 2. b) Total number of absorbed bacteria counts per area for $E$. coli on bare glass and MPN-coated substrates. Values in $a$ and $b$ represent the mean and the standard deviation $(n=3) .{ }^{*}, * *, * * *$ represent $p<0.05, p<0.01, p<0.001$, respectively. 